\title{
Multi-period pulsation and amplitude variation behavior in $\delta$ Scuti variable DQ Cephei ${ }^{\star}$
}

\author{
Z.P. $\mathbf{L i}^{1}$ and M.J. Fang ${ }^{2}$ \\ 1 Beijing Astronomical Observatory Chinese Academy of Sciences, 100012 Beijing, China \\ e-mail: lizhi@class1.bao.ac.cn \\ 2 Beijing Astronomical Observatory Chinese Academy of Sciences, 100012 Beijing, China \\ e-mail: fmj@yac.bao.ac.cn
}

Received September 22; accepted November 6, 1998

\begin{abstract}
A period analysis of the data obtained in six nights of differential photoelectric photometry of the $\delta$ Scuti star DQ Cephei (about 42 hours) confirms the pulsation modes of $f_{1}=8.0480$ cycle per day and $f_{2}=12.6826$ cycle per day. The result adjusts well to the observations that cover a time span of 46 years. Comparing with the results obtained for different years, we discovered obvious amplitude variations of the two modes. These amplitude variations show phase differences of almost 180 degrees. The variation period probably is longer than 46 years.
\end{abstract}

Key words: stars: $\delta$ Sct - oscillations - individual: DQ Cephei

\section{Introduction}

Most $\delta$ Scuti stars show multiperiodic radial or non-radial variations. Besides, many $\delta$ Scuti stars, such as $4 \mathrm{CVn}$, show strong amplitude variability of some modes from year to year, while the frequency remains essentially constant. The behavior of the amplitude variability differs from mode to mode. Even though resonances or near resonances among the excited nonradial pulsation modes of variable stars are considered as a possible cause for amplitude variation in $\delta$ Scuti variables, no satisfactory explanation or theoretical modelling have been available so far. The problem of how the amplitude varies is still open.

DQ Cephei $\left(=\right.$ HD $199908=\mathrm{BD}+55^{\circ}$ 2452), discovered by Walker in 1951 (Walker 1952), was one of the first known $\delta$ Scuti stars. In 1953, Walker proposed a period

Send offprint requests to: Z.P. Li

* Table 4 is only available in electronic form at the CDS Via anonymous http://cdsweb.u-strasbg.fr/Abstract.html of $0 \mathrm{~d} 07886$. This period is equivalent to a frequency of 12.6904 cycle per day, and was confirmed by the radial velocity study conducted by Sahade et al. (1956). Fitch organized larger scale observations in 1958 and drew the conclusion that there possibly exists two pulsation frequencies: 12.6799 and 8.0516 cycle per day (Fitch et al. 1965). In order to accurately determine the periodic variations of DQ Cephei, Pena (1983) organized a two-site photoelectric photometry collaboration in 1981. The analysis of their data provided two frequencies 8.0603 and 12.6788 cycle per day, which fits well to observations covering a time span of twenty nine years. However, in all these observations, the amplitude of the mode near 8.0603 cycle per day is small and the signal/noise ratio is not high enough to confirm its existence.

When checking the observational results, and leaving aside the mode near 8.0603 cycle per day which needs further confirmation, we find some evidences for amplitude variations of two pulsation modes during a 29 years time span. In order to identify the two pulsation modes and to study in detail the amplitude variations during a 46 years period, we organized new observations and present the results as follows.

\section{New photoelectric measurements}

The observations were made using the $85 \mathrm{~cm}$ reflector installed at the Xinglong Station of Beijing Observatory, China, with a single-channel photometer equipped with an EMI 9863 photomultiplier and controlled by a microcomputer (the photometer is the same as the one installed at the $1.58 \mathrm{~m}$ telescope in the Shanghai Observatory, Du Baitian et al. 1993). During the observations, a Johnson $V$ filter was used and the stars SAO 33038, SAO 33050 and SAO 33020 were selected as comparison stars. Within the measuring error of about $5.5 \mathrm{mmag}$, a good data set which consists of 378 data points covering about 42 hours 
Table 1. The observing Log of DQ Cephei from 3 to 8 Oct. 1997

\begin{tabular}{ccc}
\hline \hline Date (UT) & Time duration (day) & Point number \\
\hline 1997.10 .03 & 0.24 & 27 \\
1997.10 .04 & 0.36 & 88 \\
1997.10 .05 & 0.26 & 70 \\
1997.10 .06 & 0.28 & 56 \\
1997.10 .07 & 0.32 & 76 \\
1997.10 .08 & 0.28 & 61 \\
\hline \hline
\end{tabular}

Table 2. Component frequencies and amplitudes for DQ Cephei

\begin{tabular}{clll}
\hline \hline No. & Frequency $\left(\right.$ day $\left.^{-1}\right)$ & Amplitude(mag.) & Phase \\
$f_{1}$ & 12.6826 & 0.0156 & 0.9004 \\
$f_{2}$ & 8.0480 & 0.0135 & 0.8613 \\
$f_{3}$ & 1.3488 & 0.0084 & 0.9795 \\
\hline \hline
\end{tabular}

Table 3. The amplitude(magnitudes) variations of two frequencies in different years

\begin{tabular}{lllll}
\hline \hline Frequency $\left(\right.$ day $\left.^{-1}\right)$ & 1997 & 1981 & 1958 & 1951 \\
$f_{1}=12.6827$ & 0.0156 & 0.020 & 0.025 & 0.024 \\
$f_{2}=8.0480$ & 0.0135 & 0.008 & 0.006 & 0.009 \\
\hline \hline
\end{tabular}

was obtained. No evidence for any variability of the comparison stars was found. The observing Log is given in Table 1, where the date is in universal time (UT), the time duration is the length of observational run (in days) in each night, point number is the number of independent observations of DQ Cephei. The data are listed in Table 4 which is only available in electronic form at the CDS via http://cdsweb.u-strasbg.fr/Abstract.html

\section{Data analysis}

A multiple-frequency analysis of DQ Cephei was performed with a package of computer programs employing single frequency (Fourier) and multiple-frequency leastsquares techniques (program PERIOD, Breger 1990) which utilize Fourier as well as multiple-least squares algorithms. The latter technique fits a number of simultaneous sinusoidal variations in the magnitude domain and does not rely on prewhitening.

Power spectra obtained from the frequency analysis clearly show the existence of two pulsation frequencies (12.6826 cycle per day and 8.0480 cycle per day) (see Fig. 1). A third peak (1.3488 cycle per day) which is a little above the noise level can be picked up too. After removal of the first, second and third frequencies, the residuals are of the order of $0.013,0.009$ and 0.008 mmag respectively. The three-frequency solution to the data is given in Table 2 and its fits to the data are displayed in Fig. 2. Comparing the power spectrum with the previous one (Pena 1983)

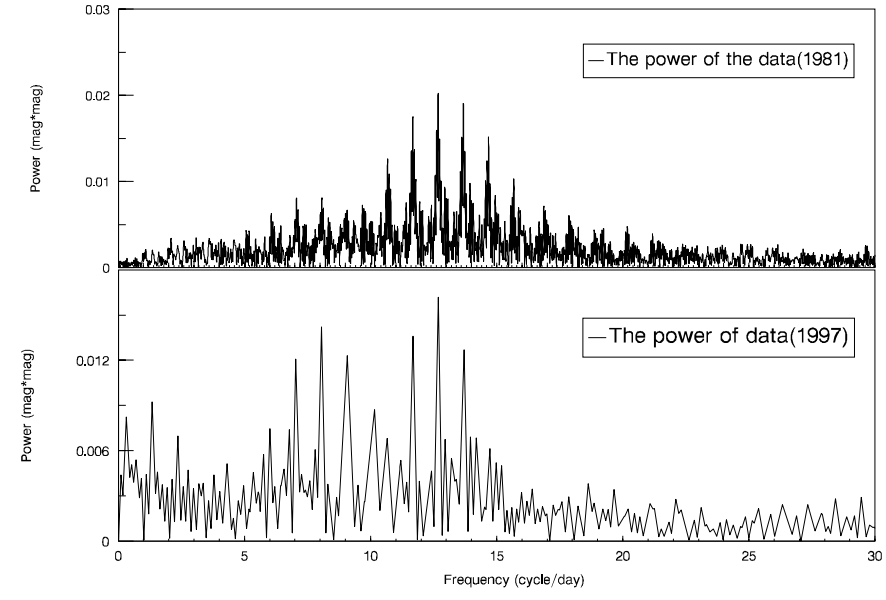

Fig. 1. The power spectra for the data observed in 1981 and 1997
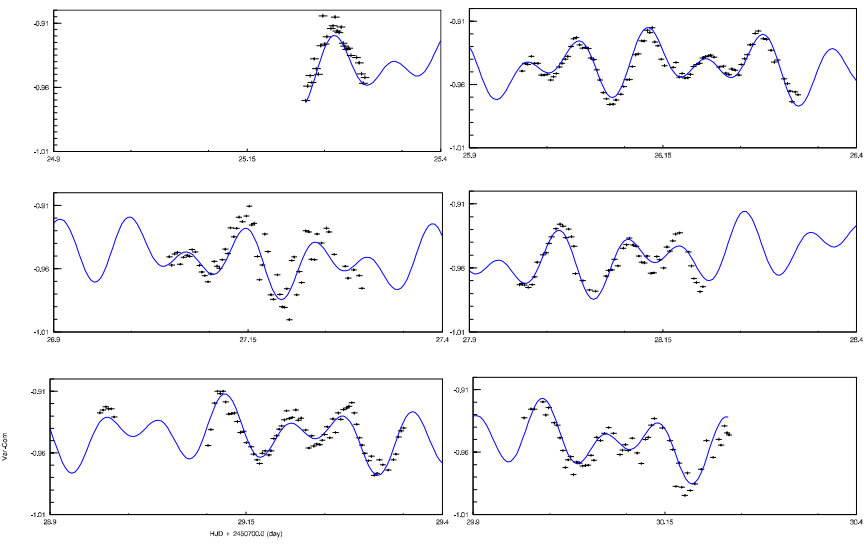

Fig. 2. The fit of the three-frequencies solution to the observations (1997)

(see Fig. 1), we find that the second peak (8.0480 cycle per day) has become significantly larger than in previous observations.

\section{Discussion}

Usually, the period analysis of the $\delta$ Scuti variables show a frequency near 1-2 cycle per day. This phenomenon is especially obvious in single-site observations and is probably due to the extinction and drift of the photometer. Therefore, we ignored the third frequency of $1.3488 \mathrm{cy}-$ cle per day, as confirmation of its existence needs further observations.

The period analysis of DQ Cephei made by Pena (1983) for different years showed some evidences for amplitude variations. Combining all those results together, we listed the amplitudes of $f_{1}$ and $f_{2}$ in different years in Table 3 and plotted them in Fig. 3. We conclude that:

1. Amplitude variations of DQ Cephei are observable during a time scale of about a decade. If such variations are periodic, the period is longer than 46 years. 


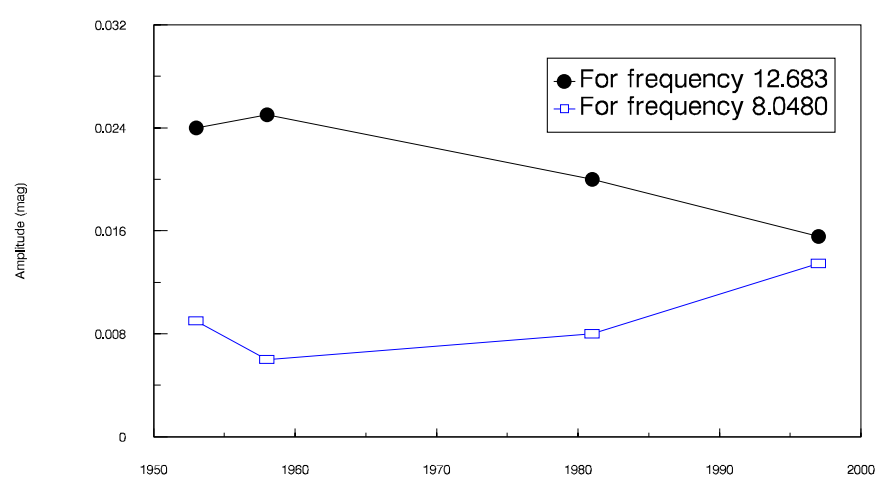

Fig. 3. Amplitude variations of two modes

2. It seems that the amplitudes of the two pulsation modes vary in opposite phases, implying energy conservation.

3. If the amplitude variations are caused by the beat of two close frequencies, the split between these frequencies should be smaller than 0.0008 cycle per day, which is too small to be explained by a rotating split. If the split is caused by the overlap of different modes, DQ Cephei should be a high evolved $\delta$ Scuti. But this does not agree with the fact that DQ Cephei is a $\delta$ Scuti variable near the main sequence.

4. The calculation made by Moskalik (1985) shows that the time scale of amplitude modulation of $\delta$ Scuti caused by resonance coupling might reach several years. This does not support the opinion that the amplitude variation of DQ Cephei can be explained by the resonance coupling. Up to now, most $\delta$ Scuti show an amplitude variation with a time scale of about a decade, which is longer than those obtained with theoretical calculations.

\section{Conclusion}

Recent observations confirm the two-frequencies solution for DQ Cephei, and the observations indicating the two pulsation modes show possible regular amplitude variations with a period exceeding 46 years. The amplitude variations seem to indicate energy conservation. The modulation period of the amplitude variations is too long to be explained by beat modulation of rotating split, and the position of DQ Cephei on HR diagram does not agree well with the hypothesis that the modulation of amplitude variation is caused by the overlap of different modes. So an interpretation in terms of two or more close frequencies can be ruled out. We suggest that the amplitude variation is caused by some kind of intrinsic amplitude variability, such as a slow transfer of pulsation energy between different modes.

Acknowledgements. This work has been supported by the Chinese National Natural Science Foundation

\section{References}

Breger M., 1990, Comm. Asteroseismology (Vienna) 20, 1

Breger M., Handler G., 1993, Comm. Asteroseismology (Vienna) 58, 1

Du Baitian, Wang Xihe, Jiang Zhaoji, et al., 1993, Ann. Shanghai Obs. 14, 192

Fitch W.S., Wehlau W.H., 1965, ApJ 142, 1616

Moskalik P., 1985, Acta Astron. 35, 3

Peña J.H., Peniche R., Margrave T.E., Hobart M.A., GonzálezBedolla S.F., 1983, A\&AS 51, 71

Sahade J., Struve O., Wilson O.C., Zebergs V., 1956, ApJ 123, 399

Walker M.F., 1952, PASP 64, 192

Walker M.F., 1953, PASP 65, 39 